\title{
DIODES AU SILICIUM UTILISÉES EN DIFFRACTION NEUTRONIQUE
}

\author{
Par L. GILLY, A. ROBERT et G. ROULT,
}

Laboratoire de Méthodes Nouvelles de Mesures de Rayonnements

et Laboratoire de Diffraction Neutronique, Centre d'Études Nucléaires de Grenoble.

Résumé. - Des détecteurs à barrière de surface ont été utilisés en diffraction neutronique :

a) Pour le positionnement optimum du cristal monochromateur et le tracé d'une carte du faisceau monochromatique.

b) Comme moniteur de comptage.

Nous avons réalisé d'une part, une microdiode qui a été positionnée en plusieurs points du canal utilisé au réacteur "Siloé " et d'autre part, une diode de plus grand diamètre qui a été montée sur le trajet du faisceau monochromatique.

Abstract. - Surface barrier detectors have been used in neutron diffraction :

a) For optimum setting of the monochromator crystal and for drawing a monochromatic beam map.

b) As a counting monitor.

We have built on the one hand a microdiode which was located in several points of the channel used at the "Siloe" reactor, and on the other hand a bigger diameter diode which was mounted in a special container in the monochromatic beam.

Introduction. - La détection des neutrons thermiques à l'aide de diodes à barrière de surface recouvertes d'une couche de produit à grande section efficace aux neutrons, remonte déjà à quelques années. Ces diodes présentent cependant l'inconvénient d'être détériorés par les neutrons rapides [1]. Il est donc préférable de les utiliser dans un faisceau de neutrons purement thermiques. Les faisceaux de neutrons monochromatiques utilisés en diffraction neutronique présentent cet avantage.

D'une part, nous avons pensé utiliser ces détecteurs comme moniteur, d'autre part, à l'aide de microdétecteurs, nous avons envisagé d'anaiyser le flux local du pinceau incident. Nous donnons, cidessous, le principe de ces diodes, leur montage dans les deux types d'utilisation, enfin leur montage électrique.
I. Description des diodes. - Les détecteurs utilisés dans le présent travail sont des détecteurs à barrière de surface, en silicium de type $n$, dopé au phosphore. La diode est recouverte d'une plage circulaire d'or, d'épaisseur voisine de $30 \times 10^{-6}$ $\mathrm{g} / \mathrm{cm}^{2}$, sur laquelle est déposé du fluorure de lithirm, d'épaisseur voisine de $10^{-4} \mathrm{~g} / \mathrm{cm}^{2}$. Le fluorure de lithium utilisé est enrichi à $92 \%$ en lithium 6 . On utilise la réaction suivante qui donne lieu à l'émission d'une particule $\alpha$ et d'un triton, après capture d'un neutron :

$$
{ }^{6} \mathrm{Li}+\mathrm{n} \rightarrow \alpha+{ }^{3} \mathrm{H}+4,787 \mathrm{MeV} .
$$

Les énergies cinétiques des particules $\alpha$ et triton sont respectivement de $2,05 \mathrm{MeV}$ et $2,74 \mathrm{MeV}$. Ces particules étant émises à $180^{\circ}$ l'une et l'autre, nous ne détecterons que l'une d'elles pour un neutron incident. 
II. Utilisation en diffraction neutronique. - Ces diodes sont utilisées à deux fins en diffraction neutronique :

10 Réglage du monochromateur et ÉtablisSEMENT DE LA CARTE DU FLUX DU FAISCEAU MONOchromatique. - Nous avons utilisé un bloc rectangulaire d'aluminium, couvrant toute la surface du canal et percé de 24 trous cylindriques de $6,5 \mathrm{~mm}$ de diamètre. Le détecteur est une microdiode carrée de 3,7 mm de côté, obtenue par découpage au jet de sable, dans une pastille de $19 \mathrm{~mm}$ de diamètre et de $0,5 \mathrm{~mm}$ d'épaisseur. La diode présente sur sa face détectrice une plage circulaire d'or de $3 \mathrm{~mm}$ de diamètre sur laquelle est situé un dépôt de fluorure de lithium de $2 \mathrm{~mm}$ de diamètre. Un fil d'or de diamètre $50 \mu$, soudé à la laque d'argent sur la couche d'or, permet d'en assurer la connexion. Une soudure à l'indium relie la face arrière du détecteur à un fil conducteur.

La diode est montée dans un cylindre de laiton de diamètre extérieur $6,4 \mathrm{~mm}$, de longueur $61 \mathrm{~mm}$ et de $0,2 \mathrm{~mm}$ d'épaisseur. Ce tube est obturé à son extrémité par une feuille d'aluminium de $0,1 \mathrm{~mm}$ d'épaisseur, assurant l'étanchéité à la lumière. Les impulsions sont reçues par un préamplificateur de charges. Pour le réglage du cristal monochromateur, on utilise deux paires de trous symétriques par rapport aux axes harizontal et vertical de la section du canal et on joue sur les deux alvéoles symétriques. Pour obtenir une carte de flux, la sonde est placée successivement dans chacun des 24 trous du bloc d'aluminium.

On dresse ensuite les courbes d'isocomptage (fig. 1).

Dans ces mesures, une chaîne type "APS 10" » est utilisée. La constante de temps de l'amplificateur est de $10^{-6} \mathrm{~s}$ et le gain de $28 \mathrm{~dB}$.

20 Utilisation COMMe moniteur. - Un bon moniteur est caractérisé par :

- un taux de comptage élevé (jusqu'à la limite de saturation de la chaîne de comptage);

- une bonne résolution en impulsions ;

- dans la mesure du possible, il doit couvrir le maximum de surface du canal sans absorber le faisceau initial ;

Les diodes au silicium recouvertes de ${ }^{6} \mathrm{LiF}$ ont ces avantages :

a) Taux de comptage élevé : Le temps mort du détecteur est de quelques nanosecondes. Les diodes décrites ci-dessous comptent environ $10^{5}$ à $1,5 \times 10^{5}$ coups/minute avec $10^{-4} \mathrm{~g} / \mathrm{cm}^{2} \mathrm{de}{ }^{6} \mathrm{LiF}$ dans un flux de $10^{6} \mathrm{n} / \mathrm{cm}^{2} / \mathrm{s}$, ce qui correspond à une efficacité d'environ $2 \times 10^{-3}$ [10]. Ce taux peut être augmenté par accroissement de la couche de ${ }^{6} \mathrm{LiF}$.

b) Bonne résolution en impulsions : Nous donnons (fig. 2) la courbe " nombre d'impulsions/seuil du sélecteur " d'une diode semblable, avec une largeur de canal de 0,5 volt.

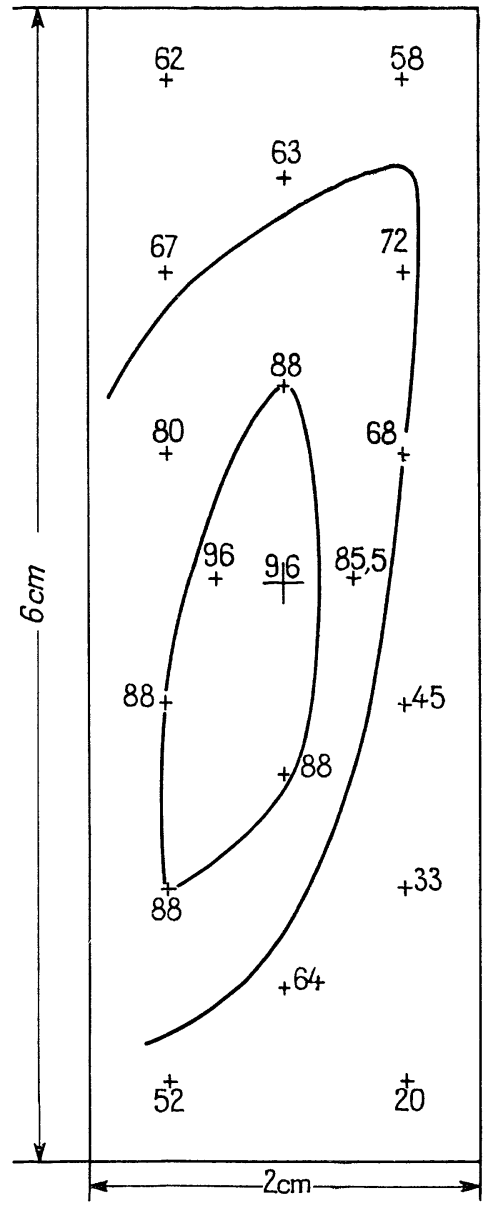

Fig. 1. - Carte de flux; les nombres qui y figurent sont des taux de comptage en unités arbitraires.

D'autre part, nous donnons la courbe en discrimination ( fig. 2). La résolution est d'autant meilleure que la couche de ${ }^{6} \mathrm{LiF}$ est mince. Dans nos conditions, elle est de $6 \%$ pour des $\alpha$ de $2 \mathrm{MeV}$ [10].

c) Les diodes actuellement utilisées ne couvrent qu'une partie du pinceau. Cependant, des comptages ont été effectués après plusieurs démontages et remontages sans donner d'écart détectable. Leur absorption des neutrons est pratiquement négligeable : coefficient d'absorption linéaire $5 \times 10^{-3} \mathrm{~cm}^{-1}$.

Ces diodes ont en plus l'avantage d'être facilement réalisables et d'être peu fragiles. La diode utilisée est une pastille de $19 \mathrm{~mm}$ de diamètre et $0,5 \mathrm{~mm}$ d'épaisseur, dorée sur une plage circulaire de $12 \mathrm{~mm}$ de diamètre. La couche de fluorure de lithium a un diamètre de $10 \mathrm{~mm}$. Le détecteur est monté dans une chambre s'adaptant sur le canal de sortie des neutrons monochromatiques. Cette chambre est obturée par de fines feuilles d'aluminium (fig. 3). Notons enfin que ces diodes ne manifestent des signes détectables de perte de résolution 


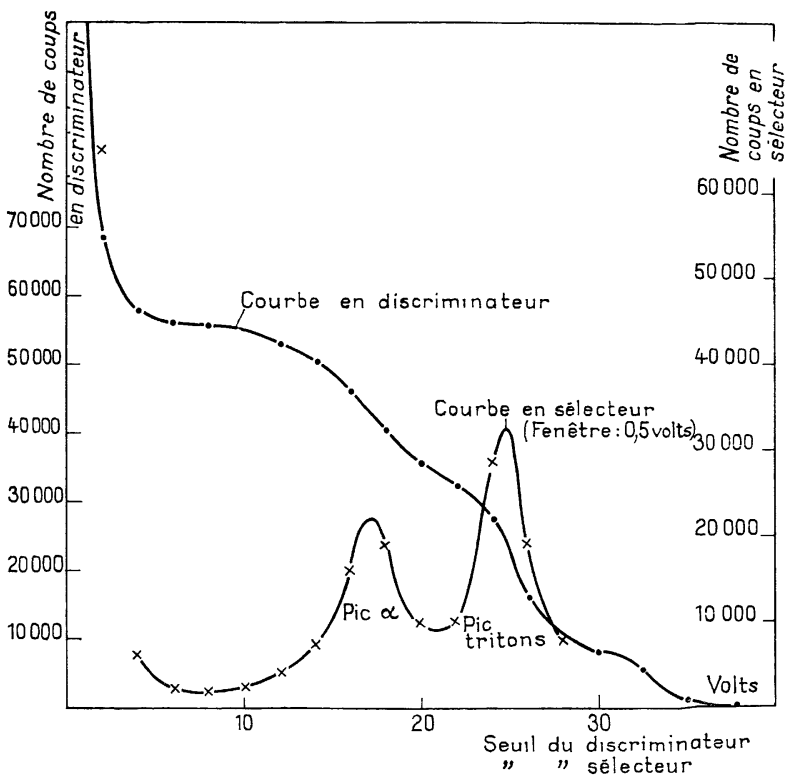

FIg. 2.

qu'après un nombre de neutrons rapides de $10^{14} \mathrm{n} / \mathrm{cm}^{2}[10]$.
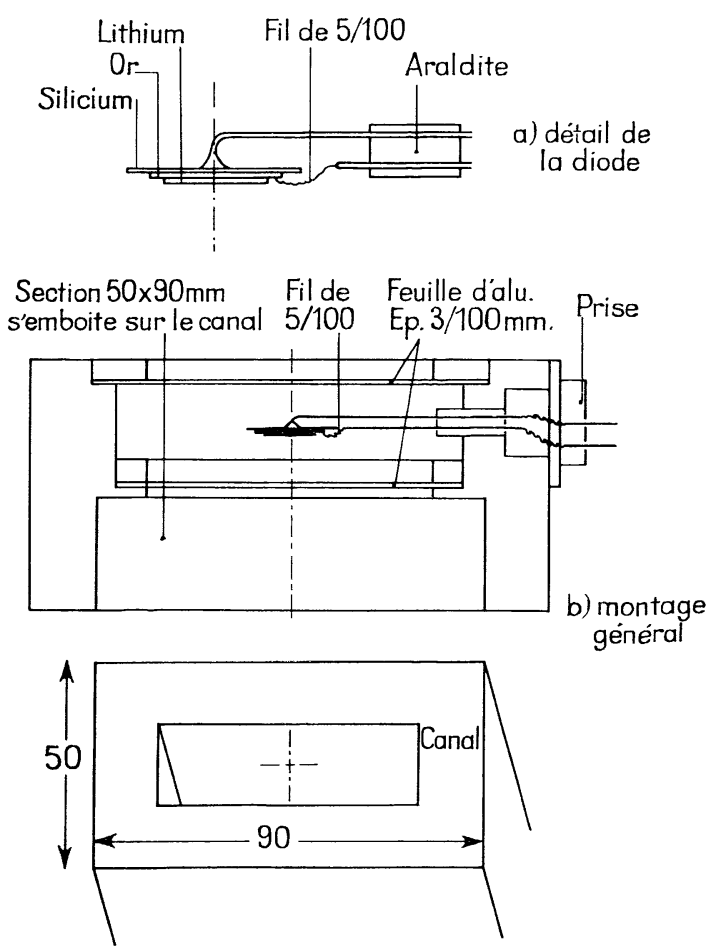

FIG. 3. - Montage mécanique.

III. Montage électrique. - 10 PrÉAmplificateur DE Charges. - A la suite du détecteur, on doit connecter un préamplificateur dit de charges. En effet, une particule incidente d'énergie $E$ dépose, par ionisation, une charge $q$ proportionnelle à $E$ dans le détecteur. Si $c$ est la capacité du détecteur, la hauteur de l'impulsion recueillie à ses bornes vaut, en tension, $v=q / c$. Elle ne serait proportionnelle à $q$ et à $E$ que si $c$ était constant.

Or, $c$ dépend essentiellement de la diode utilisée et de la tension de polarisation $V$ qui lui est appliquée $(c$ varie comme $1 / \sqrt{V})$. Il y a donc lieu d'employer un préamplificateur de charges donnant, à la sortie, des impulsions proportionnelles à $q$ et non pas à $v$. Rappelons brièvement le principe d'un tel préamplificateur [3], [5].

Un préamplificateur de charges se compose d'une part, d'un amplificateur de gain négatif $A$ (fig. 4), une capacité $C$ créant une réaction entre l'entrée et la sortie, et d'autre part d'un deuxième amplificateur de gain $A^{\prime}$. Soit $r$ l'impédance d'entrée et $t_{\mathrm{c}}$ le temps de collection des charges dans la diode; $t_{\mathrm{c}}$ est de l'ordre de quelques nanosecondes. On montre [9] que si :

$$
\begin{aligned}
& r[c+C(A+1)] \gg t_{\mathrm{c}} \\
& c / A \ll C \text { et } A \gg 1 .
\end{aligned}
$$

la hauteur de l'impulsion en $B$ vaut :

et en $S$

$$
v_{\mathrm{B}}=-q / C
$$

$$
v_{\mathrm{s}}=-A^{\prime} q I C .
$$

La hauteur de l'impulsion à la sortie $S$ du préamplificateur ne dépend plus alors de la capacité $c$ placée à l'entrée.

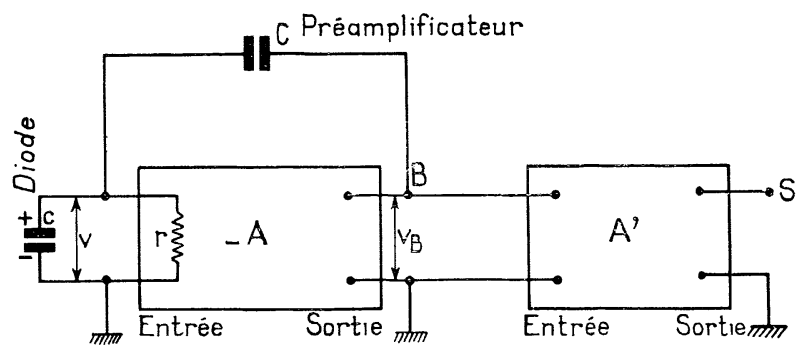

FIG. 4. - Préamplificateur : schéma synoptique.

$2^{0}$ Montage. - Il existe un ensemble d'appareils commercialisés dont l'emploi résout les problèmes d'ordre électronique. Nous avons utilisé alternativement ce matériel d'un emploi très commode et d'autres préamplificateurs [9].

La hauteur des impulsions en $S$ est donnée par (1) compte tenu du fait qu'il faut environ $3,6 \mathrm{eV}$ pour créer une charge élémentaire dans le silicium.

Le bruit électronique augmente avec la capacité $c$ placée à l'entrée du préamplificateur [3], [6]. Cette 
de la capacité $c_{p}$ du câble reliant le détecteur au préamplificateur, il y a lieu de réduire le plus possible la longueur de ce câble. Le terme $c_{d}$ diminue si l'on augmente la tension de polarisation $V$, mais alors, le courant de fuite et le bruit propre de la capacité étant la somme de celle $c_{\mathrm{d}}$ de la diode et diode augmentent aussi ; au total, le bruit observé dépend peu de la tension de polarisation [9].

Signalons, enfin, que la diode doit être placée dans un boîtier étanche à la lumière et entièrement métallique afin d'assurer un blindage parfait.

Manuscrit reçu le 7 décembre 1965.

\section{BIBLIOGRAPHIE}

[1] Dearnaley (G.), Radiation damage effects in semiconductor detectors. Nucleonics, juillet 1964, 22, 7, 78-85.

Vavilov (V. S.), Effects of radiation on semiconductors. Constants Bureau, New York, 1965.

$\mathrm{B}_{\mathrm{ABcook}}$ (R. V.), Fast neutron damage to silicon junction particle detectors. IRE Trans. Nucl. Sc., 1961, NS-8, no 1, 98.

[2] Miller (G. L.) et Gibson (W. M.), Charge collection in semiconductor radiation detectors. Nuclear Electronics, I (publication IAEA), 477.

[3] Chase (R. L.), Higinbotham (W. A.) et Miller (G. L.), Amplifiers for use with $p$-n junctions radiation detectors. IRE Trans. Nucl. Sc., 1961, NS-8, no 1, 147.

[4] Goulding (F. S.) et Hansen (W. L.), Leakage current in semiconductor junction radiation detectors and its influence on energy resolution characteristics. Nucl. Instr. and Meth., juillet 1961, 12, 2, 249.
[5] Roux (G.), Préamplificateur à faible bruit sensible à la charge, C. E. N./Saclay, Section d'Étude des Appareils d'Électronique Physique, $n^{\circ}$ DE/1579/SEAEP/205.

[6] Hahn (J.) et MaJer (R.), A low noise high gain bandwidth charge sensitive preamplifier. IRE Trans. Nucl. Sc., août 1962, NS-9, no 4, 20.

[7] Tove (P.-A.) et FALK (K.), Transit time of charge carriers in the semiconductor ionisation chamber. Nucl. Instr. and Meth., juillet 1961, 12, 2, 278.

[8] Blankenship (J.-L.) et Borkowski (C.-J.), Performance of silicon surface barrier detectors. IRE Trans. Nucl. Sc., NS-8, $\mathrm{n}^{\circ}$ 1, 17.

[9] Gilly (L.), Robert (A.) et Roult (G.), Utilisation de diodes à barrières de surface en diffraction neutronique. Rapport MNMR/DN/2 - C. E. N., Grenoble, août 1965.

[10] Fabre (R.), Utilisation de diodes du type semiconducteur comme détecteurs de neutrons. Note Techn. MNMR/1, janvier 1965. 